\title{
Variabilité spatio-temporelle des groupements végétaux d'un étang angevin (France)
}

\author{
E. Lambert-Servien 1 \\ J. Haury 2 \\ M. Guerlesquin ${ }^{1}$
}

Mots clés : étang, macrophyte, Maine-et-Loire, Analyse Factorielle des Correspondances, Classification Ascendante Hiérarchique, dynamique.

Les relations spatiales et les évolutions entre les groupements végétaux d'un étang soumis à de fortes variations de niveau ont été étudiées par des croisements manuels des relevés et par Analyse Factorielle des Correspondances. Les résultats des deux approches concordent partiellement. Deux schémas évolutifs distincts sont mis en évidence, dans la zone principale et dans la queue d'étang. De fortes disparités apparaissent en fonction d'une part, de la morphologie des rives et, d'autre part, des variations interannuelles des niveaux d'eau. Des recommandations pour une gestion patrimoniale sont présentées.

\section{Spatio-temporal variability of plant communities from a pond Anjou (France)}

Keywords : pond, macrophyte, Maine-et-Loire, Factorial Correspondence Analysis, Hierarchical Cluster Analysis, dynamics.

Spatial relationships and development in plant communities from a pond subjected to marked water level variations have been studied, using manual crossings of relevés and Factorial Correspondence Analysis. The results of these two approaches agree partially. Two distinct development schemes are presented, in the main area and in the tail-end of the pond. Large disparities appear related to the shape of the shores and to the interannual variations of water level. Advices for a natural heritage approach to management are proposed.

\section{Introduction}

De nombreux auteurs ont étudié les macrophytes des étangs français et la dynamique végétale de ces milieux (Corillion 1948 a, b, Bareau 1982, Felzines 1982, Duvigneaud 1983,-Mériaux \& Wattez 1981, Mériaux 1984, Clément \& Touffet 1987, Chaib 1992, Clément \& Bouzillé 1996). En ce qui concerne le département du Maine-et-Loire, la recherche bibliographique a souligné le manque de documents relatifs à l'inventaire

\footnotetext{
1. Laboratoire de Biologie végétale et Phytogéographie - I.R.F.A. / U.C.O., 3, place André Leroy, B.P. 808, F-49008 Angers Cedex 01, France.

2. ENSA DEERN, Ecologie \& Sciences Phytosanitaires et INRA Ecologie Aquatique, 65, rue de Saint-Brieuc, F-35042 Rennes Cedex, France.
}

actuel des milieux aquatiques (autres que la Loire) et à l'étude de leur végétation, hormis ceux de Corillion \& Guerlesquin $(1965,1969)$ et Corillion $(1975,1980$, 1994). Ceci a conduit à un travail de thèse (LambertServien 1995) pour compléter les connaissances dans ce domaine. L'étang de Péronne qui en faisait l'objet, nous a semblé pouvoir servir de référence, dans le cadre plus vaste de l'étude de la végétation des étangs et de sa dynamique, en raison de sa biodiversité phytocœnotique et de son mode de gestion. Il a permis de suivre la dynamique saisonnière sur plusieurs années, en fonction des niveaux d'eau et d'analyser l'organisation végétale ainsi que son évolution probable (à la fois au vu de la composition floristique des relevés et au vu des contacts entre groupements observés sur le terrain).

Notre objectif est de montrer qu'il existe une stratégie adaptative conduisant à une organisation végétale relativement fixe. A cette organisation s'ajoute une va- 
riabilité temporelle liée à la gestion et aux conditions climatiques, qui déterminent des périodes d'exondation. En outre, existe une colonisation erratique due à la composition floristique du bassin versant. L'étude des groupements végétaux nous conduira à l'élaboration de schémas évolutifs des communautés.

\section{Matériel et méthodes}

\subsection{Milieu d'étude}

L'étang de Péronne (37 hectares) est situé dans le massif forestier de Nuaillé-Chanteloup. Sa profondeur au centre est de l'ordre de $3 \mathrm{~m}$ et à la bonde de $5 \mathrm{~m} 40$. Les rives sont en pente douce et les atterrissements sont vaseux ou sablo-vaseux à caillouteux. L'étang est formé de deux parties, la queue étant séparée du reste de l'étang par une digue constituée de matériaux dont l'extraction a créé au fond de l'étang de petites excavations apparaissant aux basses eaux. A la queue de l'étang, où la pêche est interdite, existe une zone de reproduction naturelle avec pour corollaire une protection intégrale de la flore locale. Cet étang subit des assèchements partiels en fonction des aléas climatiques, des lâchers d'eau pour irrigation et de sa gestion piscicole et touristique.

\subsection{Données floristiques et physico-chimiques}

Le travail effectué porte sur l'ensemble des $37 \mathrm{hec}-$ tares de l'étang. Il s'est déroulé de mai à octobre durant 4 années consécutives, 1987 à 1990 . Ce travail alors étendu à d'autres étangs est actuellement poursuivi. Une campagne hivernale a complété les caractéristiques physico-chimiques.

\subsubsection{Relevés floristiques}

Ils ont été réalisés de manière à obtenir une vision la plus exhaustive possible de la végétation présente sur l'ensemble du secteur d'étude. Le principe de l'emploi de surfaces minimales, ainsi que celui des coefficients phytosociologiques d'abondance-dominance ont été retenus. Une partie des relevés a été effectuée dans les zones les plus homogènes des ceintures de végétation pour un inventaire systématique. Les autres relevés ont été positionnés selon des transects répartis sur l'ensemble de l'étang afin d'obtenir une image des gradients écologiques et des successions végétales. Les macrophytes étudiés incluent les espèces de spermatophytes, les algues des genres Chara et Nitella et les bryophytes du genre Sphagnum. La nomenclature suivie est celle de Flora europaea, de Corillion 1975 et de Touffet 1978.

\subsubsection{Caractéristiques physico-chimiques}

Les caractéristiques de l'habitat physique ont été notées une fois par mois, en moyenne, de mai à octobre, et une fois en hiver. Elles incluent la hauteur de l'eau à la bonde, la température de l'air et celle de l'eau, la nature du substrat au niveau de chacun des relevés. Des campagnes d'analyses de l'eau permettant de caractériser la qualité chimique de l'eau (conductivité, $\mathrm{pH}$, principaux nutriments minéraux), ont été réalisées avec la même fréquence. Les dosages d'éléments chimiques ont été effectués selon les normes AFNOR.

\subsubsection{Traitement des données}

La méthode des tableaux a été employée pour définir les groupements. Le statut des espèces au sein des groupements a été attribué d'après la littérature (Amicale phytosociologique 1969 , Guinochet \& Vilmorin 1973-1984, Oberdorfer 1977, Mériaux 1978, Mériaux \& Wattez 1981, Felzines 1982). La classification des relevés dans les tableaux synthétiques traduit la dynamique des groupements telle qu'elle apparaît avec les espèces relictuelles ou édificatrices.

Trois Analyses Factorielles des Correspondances (A.F.C.) et une Classification Ascendante Hiérarchique (C.A.H.) ont été utilisées pour traiter les données floristiques afin de caractériser les communautés végétales et d'établir des schémas évolutifs entre elles. Le logiciel utilisé est SPADN (C.I.S.I.A., 1991). Les résultats de ces analyses sont comparés.

\section{Résultats}

\subsection{Données physico-chimiques}

L'analyse des caractéristiques physico-chimiques, en liaison avec la flore observée, a permis de classer l'étang de Péronne dans la catégorie mésoeutrophe (Mériaux \& Wattez 1980). Les valeurs de conductivité sont comprises entre 143 et $262 \mu \mathrm{S} / \mathrm{cm}$. Le pH varie entre 7,2 et 8,8 suivant les saisons et les chaulages pour la faune piscicole. Certains pics observés pour les nitrates ont mis en évidence l'importance des techniques culturales dans le bassin versant avec apports des engrais, ainsi que l'influence du cycle hydrologique.

Toutefois, le suivi effectué n'a pas permis de mettre en évidence des éléments prouvant des modifications de la flore macrophytique en place hormis pour les characées. Le maintien de 2 espèces en 1989 et 1990 : Chara fragifera et $C$. delicatula, parmi les 9 recensées en 1988 (Tableau 1) est aussi lié à des phénomènes d'eutrophisation dus au phosphore auxquels s'ajoutent des conditions climatiques (températures élevées) et hydrologiques (baisse du niveau de l'étang) qui ont contribué à une exondation importante ou à un réchauffement des sites de développement des characées. Une différenciation est faite en fonction des dominances pour identifier des communautés à characées appartenant aux Charetea. Ces communautés semblent 





assez instables dans le temps et il est difficile d'identifier précisément de réel groupement à partir d'un nombre relativement faible de relevés. Elles se maintiennent parfois sous des strates phanérogamiques d'où la présence d'un faciès à Juncus heterophyllus (Tableau 1).

\subsection{Traitements manuels}

Les résultats des traitements manuels mettent en évidence la diversité phytocœnotique de l'étang où s'observent 28 groupements ou faciès. Ils se retrouvent, pour la majorité d'entre eux, avec une large répartition française (Rallet 1936, Corillion 1948 a, b, Mériaux 1978, Mériaux \& Wattez 1981, Felzines 1981, 1982, Bareau 1982). Ces ensembles, caractérisés par leur espèce dominante, appartiennent aux groupements d'eau profonde ou de pleine eau : Nuphar lutea, Nymphaea alba, Potamogeton lucens, Chara sp. ou Nitella sp. : Chara braunii, C. delicatula, C. fragifera, C. globularis, Nitella confervacea, $N$. flexilis, $N$. gracilis, $N$. opa$\mathrm{ca}, \mathrm{N}$. translucens, (certains ensembles à characées appartiennent aussi aux végétations de bordure), Myriophyllum spicatum, Potamogeton natans, Potamogeton gramineus. D'autres groupements participent à la transition entre les sites de pleine eau et ceux des bordures: Ranunculus aquatilis, Polygonum amphibium, Elatine hexandra, Eleocharis palustris, Hottonia palustris, Littorella uniflora (amphiphyte dont le cortège floristique s'étend jusqu'à la périphérie de l'étang). Les ensembles végétaux caractérisés par les espèces suivantes s'étendent des bordures jusqu'aux ceintures périphériques : Eleocharis acicularis, Rorippa amphibia et Oenanthe aquatica, Scirpus lacustris, Phragmites australis, Typha latifolia, Sparganium erectum, Carex elata. Les groupements à Carex pseudo-cyperus, $\mathrm{Ca}$ rex vesicaria, Juncus bulbosus, Juncus heterophyllus, Phalaris arundinacea, ne participent qu'à la composition des ceintures périphériques. Les ensembles à $\mathrm{Mo}$ linia caerulea et Salix atrocinerea sont rarement inondés. Un groupement à Eleocharis ovata est apparu de manière tout à fait exceptionnelle.

\subsection{Analyses multivariées (Tableau 2)}

- La première A. F. C. a été effectuée pour mettre en évidence les relations entre les relevés et les ensembles floristiques, sans distinction a priori des taxons végétaux. Elle présente une structure hexadimensionnelle. L'importance des espèces des ceintures extérieures boisées apparaît dès le premier axe. Les ensembles végétaux (plantes herbacées de milieux assez secs ou herbacées pionnières, fixatrices d'azote, ...) apparaissant sur les 4 axes suivants, sont liés à la particularité de cet étang dont les rives sont en pente douce et sou- vent exondées. La nette différence de milieu et de gestion entre la queue de l'étang et la partie principale est exprimée par le sixième axe.

- La seconde A. F. C. a été réalisée pour préciser les relations entre les espèces des groupements aquatiques et hydrophiles. Le premier axe traduit la fermeture progressive du milieu, typique de la dynamique naturelle d'un étang. Dès le second axe apparaît la différence spatiale entre la partie principale de l'étang marquée par les groupements étendus à Littorella uniflora et les végétations à Characées, et la queue de l'étang où dominent les hygrophytes et hélophytes de milieux mésotrophes à eutrophes : Hottonia palustris, Rorippa amphibia, Oenanthae aquatica, Alopecurus aequalis, A. geniculatus. Le troisième axe différencie les groupements de bordure où dominent les hélophytes avec la présence d'un faciès à Ranunculus lingua. L'axe 4 est marqué par trois groupements particuliers à : Elatine hexandra, Eleocharis ovata, ou Juncus heterophyllus. Enfin, cette seconde A.F.C. souligne avec le cinquième axe la différence entre la rive sud de la partie principale de l'étang où domine le groupement à Littorella uniflora et la rive nord où se développent de nombreux ensembles à characées.

- La troisième A. F. C. ne concernait que les espèces aquatiques et les hélophytes. Le facteur répartition spatiale apparaît alors dès le premier axe. L'opposition entre les hélophytes et les hydrophytes caractérise le second axe sur lequel les relevés et les espèces indiquent la dominance des hélophytes dans la queue de l'étang et sur la rive nord de la partie principale.

La C. A. H. associée à cette dernière analyse (Fig. 1) a permis de différencier 18 classes. Plusieurs d'entre elles correspondent à des communautés ou groupements distingués aussi manuellement dont les espèces caractéristiques sont : Hottonia palustris, Phragmites australis, Molinia caerulea, Potamogeton lucens, Littorella uniflora, Chara sp., Eleocharis ovata. Mais plusieurs classes apportent un complément d'information soulignant les diverses évolutions végétales en fonction des emplacements (rive nord ou sud, queue d'étang). Quelques petits faciès non retenus dans l'étude par tableau apparaissent ici : faciès à Carex pseudocyperus ou à Elodea canadensis.

\section{Discussion}

Certains ensembles végétaux se rapprochent des végétations décrites par les auteurs en diverses régions, parmi lesquels ceux à Chara et Nitella sp. (Corillion 1949) (Tableau 1), les groupements à Littorella uniflo$r a$ et à Scirpus lacustris (Felzines 1982), les végétations à Elatine hexandra, ou à Eleocharis acicularis 
Tableau 2. Résultats des trois Analyses Factorielles des Correspondances traduisant l'organisation des phytocœnoses de l'étang de Péronne. Table 2. Results of the three Factorial Correspondence Analysis showing the phytocœnitic organisation of the pond at Peronne.

\begin{tabular}{|c|c|c|}
\hline $\begin{array}{c}\text { Analyse Factorielle des Correspondances } \\
\mathbf{n}^{\circ} \mathbf{1} \\
439 \text { relevés }-218 \text { taxons }\end{array}$ & $\begin{array}{c}\text { Analyse Factorielle des Correspondances } \\
\qquad \mathbf{n}^{\circ} \mathbf{2} \\
430 \text { relevés }-198 \text { taxons }\end{array}$ & $\begin{array}{c}\text { Analyse Factorielle des Correspondances } \\
\mathbf{n}^{\circ} \mathbf{3} \\
396 \text { relevés }-97 \text { taxons }\end{array}$ \\
\hline $\begin{array}{c}6 \text { axes significatifs } \\
\text { Inertie cumulée : } 17 \%\end{array}$ & $\begin{array}{c}5 \text { axes significatifs } \\
\text { Inertie cumulée : } 14 \%\end{array}$ & $\begin{array}{c}2 \text { axes significatifs } \\
\text { Inertie cumulée : } 8 \%\end{array}$ \\
\hline $\begin{array}{l}\text { Signification écologique des axes : } \\
\text { 1.Espèces ligneuses des ceintures } \\
\text { extérieures: Cornus sanguinea, Prunus avium, } \\
\text { Pinus pinaster... } \\
\text { Hydrophytes : Nitella opaca, Polygonum } \\
\text { minus, Potamogeton lucens, Myriophyllum } \\
\text { spicatum... } \\
\text { 2. Herbacées : Trifolium repens, Geranium } \\
\text { robertianum, Fumaria officinalis, Lysimachia } \\
\text { nummularia. } \\
\text { Espèces de boisements : Cornus sanguinea, } \\
\text { Prunus avium, Stachys officinalis ... } \\
\text { 3. Herbacées de milieux assez secs : } \\
\text { Taraxacum officinale, Arenaria trinervia, Agrimonia } \\
\text { eupatoria .... } \\
\text { Herbacées sur vases humides enrichies : } \\
\text { Polygonum minus, Bidens tripartita, Trifolium } \\
\text { repens, Lotus uliginosus ... } \\
\text { 4. Herbacées pionnières fixatrices d'azote : } \\
\text { Lotus uliginosus, Trifolium arvense, T. repens, T. } \\
\text { pratense ... } \\
\text { Herbacées annuelles sur vases enrichies : } \\
\text { Polygonum minus, Bidens tripartita ... } \\
\text { 5. Héliophytes -Plantes de sous-bois. } \\
\text { 6. Zone principale de I'étang : Littorella } \\
\text { uniflora, Nitella opaca, Chara globularis ... } \\
\text { Queue d'étang : Hottonia palustris, } \\
\text { Alopecurus aequalis, Alopecurus geniculatus, } \\
\text { Ranunculus aquatilis, Oenantha aquatica ... }\end{array}$ & $\begin{array}{l}\text { Signification écologique des axes : } \\
\text { 1. Variation temporelle et assèchement } \\
\text { Hydrophytes : Nitella opaca, Potamogeton } \\
\text { lucens, Hottonia palustris, Nitella confervacea ... } \\
\text { Groupements à Molinia caerulea et Salix } \\
\text { atrocinerea, Populus tremula } \\
\text { 2. Variation spatiale } \\
\text { Queue d'étang : Hottonia palustris, Rorippa } \\
\text { amphibia, Oenanthe aquatica, Alopecurus aequalis, } \\
\text { A. geniculatus ... } \\
\text { Zone principale de l'étang : Littorella uniflora, } \\
\text { Chara globularis, Nitella opaca ... } \\
\text { 3. Groupements de bordure : Ranunculus } \\
\text { lingua, Phragmites australis, Phalaris arundinacea, } \\
\text { Myosotis secunda ... } \\
\text { Groupements exceptionnellement } \\
\text { inondés : Carex hirta, Molinia caerulea,Salix } \\
\text { atrocinerea ... } \\
\text { 4. Groupements particuliers : } \\
\text { Elatine hexandra } \\
\text { Eleocharis ovata } \\
\text { Juncus heterophyllus } \\
\text { 5. Pelouses à Littorella uniflora ou prairies } \\
\text { infraaquatiques à Characées, dans la zone } \\
\text { principale de l'étang }\end{array}$ & $\begin{array}{l}\text { Signification écologique des axes : } \\
\text { 1. Zone principale de l'étang : Juncus } \\
\text { acutiflorus, Molinia caerulea, Littorella uniflora, } \\
\text { Scutellaria galericulata ... } \\
\text { Queue d'étang :Hottonia palustris, Rumex } \\
\text { crispus, Alopecurus aequalis, A. geniculatus, } \\
\text { Ranunculus aquatilis } \\
\text { 2. Hydrophytes : Chara globularis, Littorella } \\
\text { uniflora, Myriophyllum alterniflorum, Chara } \\
\text { delicatula ... } \\
\text { Hélophytes et hémicryptophytes : } \\
\text { Phragmites australis, Phalaris arundinacea, } \\
\text { Myosotis secunda, Molinia caerulea, Calystegia } \\
\text { sepium, ... }\end{array}$ \\
\hline
\end{tabular}

(Szmeja \& Clément 1990). Dans les étangs angevins, les végétations des bordures sont localement pénétrées, ou dominées, par Juncus heterophyllus (Tableau 3) rare amphiphyte à répartition géographique ouestméditerranéenne atlantique, parvenant en Anjou et dans le sud-armoricain à proximité de sa limite septentrionale. Cette juncacée est absente dans les descriptions phytosociologiques régionales consultées. Seul un groupement à Juncus heterophyllus, caractéristique des milieux oligotrophes, est décrit en Bretagne par Clément \& Touffet (1987) et l'espèce est citée comme caractéristique des Littorelletea et de l'HydrocotyloBaldellion par Szmeja \& Clément (1990). Les végétations à Juncus heterophyllus se développent dans le cadre des potamaies de bordures ou des prairies à Littorella uniflora, submergées une grande partie de l'année, auxquelles succèdent les groupements à Eleocharis palustris ou à Eleocharis acicularis, ordinairement à l'écart des grands hélophytes (rives et grèves à faible déclivité). Elles se différencient accessoirement aux dépens des myriophyllaies et prairies de Characées. Observé en pleine eau ou sur des biotopes exondés, le groupement présente des faciès mono ou polyspécifiques plus ou moins denses.

L'examen du tableau 3 montre le développement par place d'un faciès à Alisma plantago-aquatica. Le stade final de l'évolution du groupement à Juncus heterophyllus, par sédimentation active et baisse corrélative des niveaux d'eau, conduit à la formation d'ensembles dominés par les éléments des phragmitaies et scirpaies des ceintures périphériques. Le groupement à Hottonia palustris (Tableau 4) observé en queue d'étang, dans une petite dépendance riche en matière organique, soumise à d'importantes variations de niveau d'eau, ne peut être décrit qu'à partir de deux étangs angevins sur une soixantaine, parcourus. Le biotope et les valeurs de conductivité sont comparables à ce qui est présenté par Chaib (1992) ; le pH est plus basique : 6,6 à 7,8. Le groupement se développe en bordure de la saulaie ripariale. Les espèces des Potametea (présence impor- 




Fig.1. Distribution dans le plan factoriel $\mathrm{F} 1 \mathrm{xF} 2$ des 18 classes de relevés identifiées par C.A.H. : groupements et dynamique de la végétation. Fig.1. Distribution of the 18 clusters of «relevés» within the F1xF2 factorial plane of H.C.A.: groups and vegetation dynamics. 
Tableau 3. Groupement à Juncus heterophyllus (étang de Péronne, Maine-et-Loire).

Table 3. Juncus heterophyllus community of the pond at Péronne( Maine-et-Loire).

\begin{tabular}{|c|c|c|c|c|c|c|c|c|c|c|c|c|c|c|c|}
\hline Numéro des relevés & P179 & P186 & P178 & P164 & P137 & P148 & $\mathrm{P} 134$ & P150 & P146 & P176 & P193 & P189 & P162 & $\mathrm{P} 144$ & Classe \\
\hline Surface (en m2) & $\mathrm{E} 40$ & $\mathrm{E} 20$ & $\mathrm{E} 40$ & $\mathrm{SH}$ & SH & $\mathrm{SH}$ & $\mathrm{SD}$ & SD & $\mathrm{SD}$ & SS & E15 & E15 & $\mathrm{SH}$ & $\mathrm{SD}$ & de \\
\hline Eau; Substrat Détrempé; Sub.Humide; Sub.Sec & 8 & 20 & 8 & 9 & 16 & 10 & 30 & 16 & 12 & 4 & 6 & 4 & 6 & 16 & Présence \\
\hline Substrat:Cailloux; Sable; Sable/Vase; Vase & SV & SV & SV & SV & SV & $\mathrm{V}$ & V & V & $\mathrm{V}$ & V & SV & SV & SV & SV & \\
\hline \multicolumn{16}{|l|}{ Caractéristique d'association } \\
\hline Juncus heterophyllus Dufour & 2.2 & 4.4 & 2.2 & 3.3 & 2.2 & 4.4 & 4.4 & 4.4 & 2.2 & 3.3 & 4.4 & 2.2 & 2.2 & 4.4 & $\mathrm{~V}$ \\
\hline \multicolumn{16}{|l|}{ Espèces des Littorelletea } \\
\hline Littorella uniflora (L.) Ascherson & 2.2 & + & 1.1 & + & 1.1 & 1.1 & & & & & & & & & III \\
\hline Hydrocotyle vulgaris $\mathrm{L}$. & & & & 2.2 & + & & & & & & + & & & & II \\
\hline Baldellia ranunculoides (L.) Parl. & & & & & + & 1.1 & & & & & & & & & I \\
\hline Eleocharis acicularis (L.) Roemer et Schultes & & & & & & & & & + & 1.1 & & & & & I \\
\hline Alopecurus aequalis Sobol. & & & & & & & 1.1 & & & & & & & & I \\
\hline Elatine hexandra (Lapierre) DC. & & & & & & + & & & & & & & & & I \\
\hline \multicolumn{16}{|l|}{ Caractéristiques des Potametea } \\
\hline Nymphaea alba $L$. & & & & + & & & 1.1 & & + & & & & + & & II \\
\hline Potamogeton natans $\mathrm{L}$. & & 1.1 & & & & + & & & & & + & + & & & II \\
\hline Polygonum amphibium $\mathrm{L}$. & & & + & & & & + & & & & & + & & & II \\
\hline Potamogeton crispus L. & & + & + & & & & & & & & & & & & I \\
\hline Nuphar lutea (L.) Sibth. \& Sm. & & & & & + & & & + & & & & & & & I \\
\hline Myriophyllum spicatum L. & & & 1.1 & & & & & & & & & & & & I \\
\hline Utricularia australis R. Br. & & 1.1 & & & & & & & & & & & & & I \\
\hline Hydrocharis morsus-ranae $\mathrm{L}$. & + & & & & & & & & & & & & & & I \\
\hline Potamogeton lucens L. & & & + & & & & & & & & & & & & I \\
\hline Ranunculus aquatilis $\mathrm{L}$. & & & + & & & & & & & & & & & & I \\
\hline \multicolumn{16}{|l|}{ Caractéristiques des Phragmitetea } \\
\hline Alisma plantago-aquatica $\mathrm{L}$. & & + & 1.1 & + & + & 2.2 & + & 3.3 & & + & + & + & & + & IV \\
\hline Oenanthe aquatica (L.) Poiret & & & & & + & + & + & & 2.2 & & & & & + & II \\
\hline Lythrum salicaria L. & & & & 1.1 & 1.1 & & + & & + & & & & & & II \\
\hline Rorippa amphibia (L.) Besser & & & 1.1 & & & & + & & + & & + & & & & II \\
\hline Phalaris arundinacea $\mathrm{L}$. & & & 1.1 & & 1.1 & & & & & & & + & & & II \\
\hline Eleocharis palustris (L.) Roemer \& Schultes & & & 1.1 & 1.1 & & + & & & & & & & & & II \\
\hline Scirpus lacustris L. & & & & 4.4 & & & & + & & & & & + & & II \\
\hline Galium palustre $\mathrm{L}$. & & & & & 1.1 & & & & & & + & + & & & II \\
\hline Mentha aquatica $\mathrm{L}$. & & & & & + & & & & & & + & + & & & II \\
\hline Phragmites australis(Cav.) Trin. & & & & & & & & & 2.2 & + & & & & & I \\
\hline Iris pseudacorus $\mathrm{L}$. & & & & & & 1.1 & & & & & & & & & I \\
\hline Veronica scutellata L. & & + & & & & & & & & & & & & & I \\
\hline Carex vesicaria $L$. & & & & & & & & & & & & + & & & I \\
\hline \multicolumn{16}{|l|}{ Autres espèces } \\
\hline Ranunculus flammula L. & & + & & 1.1 & 1.1 & & + & & 1.1 & & & + & & & III \\
\hline Polygonum persicaria L. & & & & & & + & & + & & & & & & 1.1 & II \\
\hline Glyceria plicata (Fries) Fries & & & + & & 1.1 & & & & & & & & & & I \\
\hline Epilobium tetragonum L. & & & & & + & & & & & & & & & & I \\
\hline Eleocharis ovata (Roth) Roemer \& Schultes & & & & & & + & & & & & & & & & I \\
\hline
\end{tabular}

tante de Ranunculus aquatilis) et des Littorelletea participant à sa constitution, sont parfois différentes de celles présentées par Chaib, 1992. Mais il est peu à peu envahi par les éléments des Phragmitetea contribuant à son élimination. Si l'espèce est assez fréquente dans la vallée moyenne de la Loire et dans le Bas-Allier (Felzines \& Loiseau 1990), elle est en forte régression sur l'ensemble de son aire orientale (Chaib 1992).

Les résultats obtenus ont permis de distinguer plusieurs niveaux d'évolution dans l'approche dynamique du site et de proposer deux schémas évolutifs (Fig. 2).
Ces derniers traduisent à la fois les particularités de l'étang étudié mais aussi des phénomènes généraux liés aux stratégies adaptatives, aux compétitions interspécifiques, au stock de semences contenues dans les vases et aux apports extérieurs.

Ces schémas décrivent de nombreuses successions végétales observées par les auteurs (Mériaux 1978, Géhu \& Mériaux 1981, Haury 1991, Chaib 1992) et montrent une constante propre aux milieux aquatiques régionaux ou même ouest européens : la relative homogénéité des milieux permet le maintien de la plupart 
Tableau 4. Groupement à Hottonia palustris de l'étang de Péronne, (Maine-et-Loire).

Table 4. Hottonia palustris community of the pond at Péronne (Maine-et-Loire).

\begin{tabular}{|c|c|c|c|c|c|c|c|c|c|c|c|}
\hline Numéro des relevés & P334 & P300 & $\mathrm{P} 250$ & P200 & P340 & P343 & P352 & P355 & P350 & $\mathrm{P} 217$ & Classe \\
\hline Surface (en $\mathrm{m} 2$ ) & 2 & 6 & 6 & 2 & 2 & 2 & 4 & 8 & 8 & 12 & de \\
\hline Eau; Substrat Détrempé; Sub.Humide; Sub.Sec & E30 & E10 & E15 & E25 & E30 & E30 & E5 & SS & SH & SH & Présence \\
\hline Substrat:Cailloux; Sable; Sable/Vase; Vase & SV & SV & SV & SV & SV & SV & $\mathrm{V}$ & $\mathrm{V}$ & $\mathrm{V}$ & $\mathrm{V}$ & \\
\hline \multicolumn{12}{|l|}{ Caractéristique d'association } \\
\hline Hottonia palustris $\mathrm{L}$. & 3.3 & 2.2 & 1.1 & 1.1 & 1.1 & 2.2 & 2.2 & 1.1 & 1.1 & + & $\mathrm{V}$ \\
\hline \multicolumn{12}{|l|}{ Caractéristiques des Potametalia } \\
\hline Ranunculus aquatilis $\mathrm{L}$. & 1.1 & 2.2 & 3.3 & 1.1 & + & + & & & & & III \\
\hline Callitriche stagnalis Scop. & & & & 1.1 & & & & & & & I \\
\hline Elodea canadensis Michx & & + & & + & & & & & & & I \\
\hline Potamogeton pectinatus $\mathrm{L}$. & & & & & & & & + & + & & I \\
\hline Potamogeton natans $\mathrm{L}$. & & + & & & & & & & & & I \\
\hline \multicolumn{12}{|l|}{ Caractéristiques des Littorelletea } \\
\hline Juncus heterophyllus Dufour & & 1.1 & & & & & + & & & & I \\
\hline Baldellia ranunculoides (L.) Parl. & & & & & & & + & & & 1.1 & I \\
\hline Hydrocotyle vulgaris $\mathrm{L}$. & & & + & & & & & & & & I \\
\hline \multicolumn{12}{|l|}{ Caractéristiques des Phragmitetea } \\
\hline Oenanthe aquatica (L.) Poiret & + & 1.1 & 1.1 & & 1.1 & + & + & & & + & IV \\
\hline Alisma plantago-aquatica $\mathrm{L}$. & + & & & & + & & + & 1.1 & & 1.1 & III \\
\hline Eleocharis palustris (L.) Roemer \& Schultes & & & + & & & & & + & + & & II \\
\hline Glyceria plicata (Fries) Fries & & & & & & & & 1.1 & 1.1 & & I \\
\hline Rorippa amphibia (L.) Besser & & + & + & & & & & & & & I \\
\hline Scirpus lacustris $\mathrm{L}$. & & & & & & & 2.2 & & & & I \\
\hline Typha angustifolia $L$. & & & & & & & & & & 1.1 & I \\
\hline Lythrum salicaria $L$. & & + & & & & & & & & & I \\
\hline Phragmites australis (Cav.) Trin. & & & + & & & & & & & & I \\
\hline Sparganium erectum $\mathrm{L}$. & & & & & & & & & & + & I \\
\hline \multicolumn{12}{|l|}{ Autres espèces } \\
\hline Ranunculus flammula $\mathrm{L}$. & & + & & & & & + & & & 1.1 & II \\
\hline Alopecurus aequalis Sobol. & & & & & & + & + & 1.1 & & & II \\
\hline Oenanthe crocata $\mathrm{L}$. & & & & & & & & 1.1 & 1.1 & & I \\
\hline Alopecurus geniculatus $\mathrm{L}$. & & & 1.1 & & & & & & & & I \\
\hline
\end{tabular}

des groupements dans de nombreux étangs. Mais ces schémas soulignent une particularité de l'étang de Péronne, en relation avec ses caractéristiques physicochimiques et son mode de gestion : une richesse des groupements (28) appartenant à des ceintures végétales différentes.

Le premier schéma met en évidence la diversité floristique et la dynamique de la partie principale de l'étang. Si l'ensemble de ce schéma a pu être observé sur la rive nord, avec maintenant d'importantes surfaces recouvertes par les hélophytes et bordées par la saulaie ripariale, il n'en est pas de même sur la rive opposée. Cette dernière présente une évolution, qui pour la majeure partie de la rive, en est encore au stade du groupement à Littorella uniflora. Le schéma concernant la queue de l'étang permet de constater : - l'absence des groupements d'eau profonde, - l'installation d'ensembles végétaux différents de ceux observés dans la partie principale, - l'évolution végétale beaucoup plus avancée de cette partie de l'étang.

La dynamique du site apparaît nettement au vu de ces schémas évolutifs. Les deux premiers niveaux d'évolution expriment une dynamique à long terme, propre aux étangs en général : 1) colonisation par les grands hélophytes ; 2) atterrissement dans la queue de l'étang, très riche en matière organique et souvent exondée, présence de nombreuses végétations à hélophytes et installation de la saulaie. 
Dynamique de la végétation dans la partie principale de l'étang
Dynamique de la végétation dans la queue d'étang

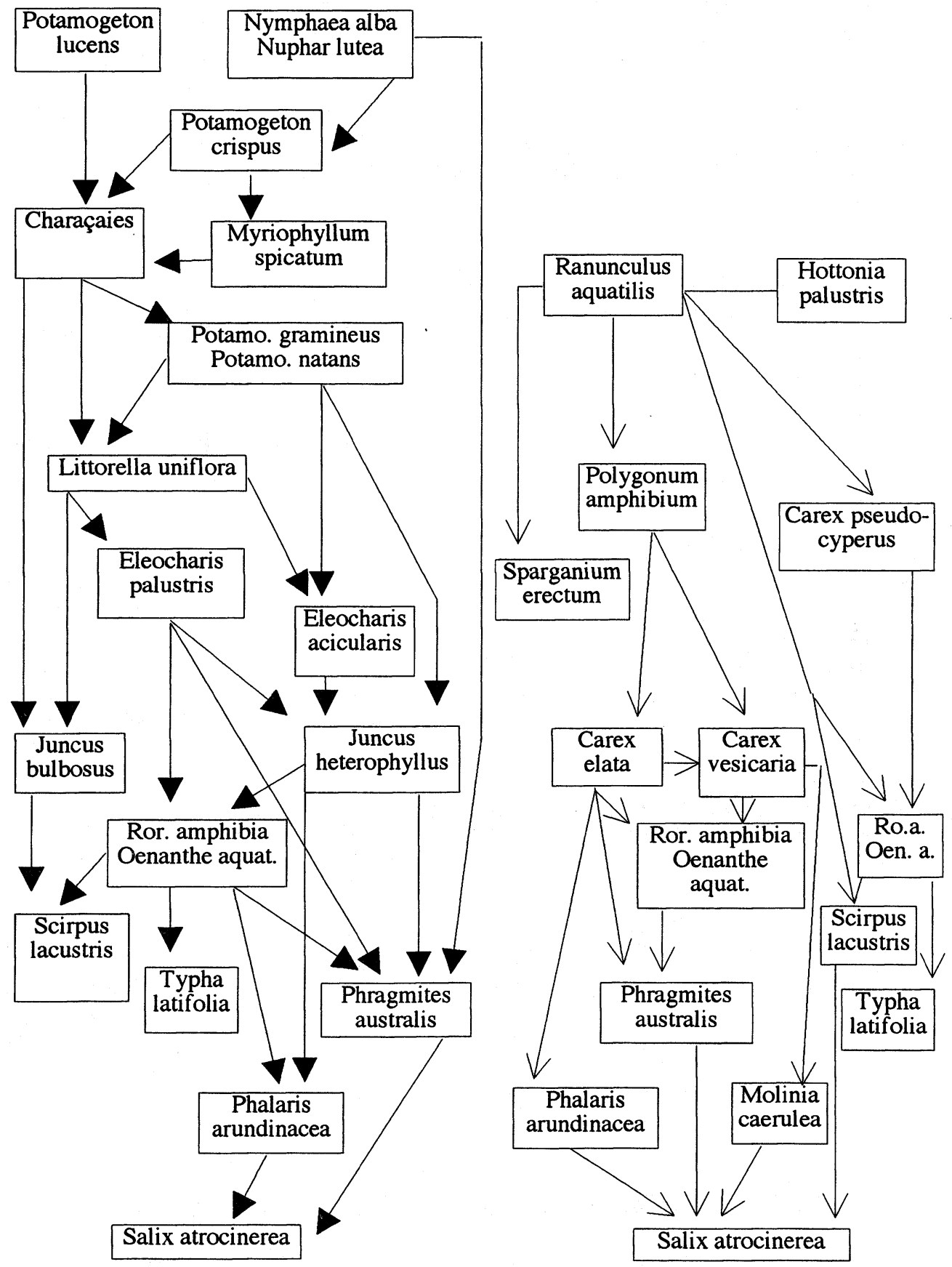

Fig. 2. Schémas évolutifs de la végétation de l'étang de Péronne (Maine-et-Loire).

Fig 2. Development trends of the vegetation of the pond of Péronne (Maine-et-Loire). 
Deux autres niveaux d'évolution à déterminisme saisonnier sont propres à cet étang. Ils sont relatifs à l'apparition de groupements sporadiques de thérophytes dans la partie principale de l'étang (celui à Eleocharis ovata p. ex.) ou au développement de groupements particuliers dans les dépendances restant en eau lors des assèchements (groupements à characées ou à Hottonia palustris). Les différences climatiques et celles liées à la gestion des niveaux d'eau de l'étang au cours des années de suivi ont donc contribué à une extension variable des groupements végétaux, soulignant quelques similitudes entre les années 1987 et 1989 caractérisées par une période de sécheresse lors du développement de la végétation. L'année 1988, beaucoup plus humide et l'année 1990 , plus sèche mais au cours de laquelle la bonde de l'étang a été maintenue fermée afin d'éviter une baisse importante des niveaux d'eau, présentaient aussi quelques similitudes.

Les variations interannuelles des niveaux d'eau déterminent des espèces et des groupements végétaux différents d'une année à l'autre, n'ayant pas tous la même valeur patrimoniale. Elatine hexandra a été observée chaque année à l'état sporadique. Cette espèce se développant au ras du sol, se voit souvent recouverte par la strate supérieure à Baldellia ranunculoides ou Alisma plantago-aquatica. Elle se développe sur milieux vaseux, exondés ou non, en association ou en concurrence avec des espèces appartenant aux Littorelletea ou aux Phragmitetea. Eleocharis ovata est une espèce épisodique qui n'a pas été vue depuis de nombreuses années et qui a été proposée par Corillion (1992) dans ses propositions pour une liste rouge d'espèces indigènes à protéger. Espèce vulnérable, elle s'est développée à la faveur d'une baisse importante des eaux, grâce au pouvoir considérable de conservation de ses graines. Elle est citée en Bretagne par Szmeja et Clément (1990) dans l'ensemble des Littorelletea; Lhote \& Shaefer (1983) mentionnent un Eleocharetum ovatae fragmentaire, végétation pionnière de vases exondées, dans un étang asséché durant plus d'un an, aux vases argileuses et humifères. Parmi les espèces observées au cours de cette étude, retenons enfin : Hippuris vulgaris, Juncus anceps (espèces protégées au niveau régional des Pays de Loire), Ranunculus lingua, Gratiola officinalis (espèces protégées au niveau national), Luronium natans (citée dans la Convention de Berne et la Directive communautaire), Ranunculus ololeucos et $R$. lingua, Potamogeton pectinatus, Hypericum elodes, Hippuris vulgaris, Eleocharis ovata, (plantes menacées en Anjou, Corillion 1992).
Il faudrait à tout prix maintenir une diversité de gestion des niveaux d'eau et, si possible, favoriser des baisses de niveau estivales pour garantir l'intérêt patrimonial du site et non contribuer à le dégrader. Les variabilités interannuelles sont importantes pour les végétations comme celles à Eleocharis ovata. Les baisses seraient à moduler puisque la baisse des eaux et le dessèchement prononcé de la vase peuvent éliminer peu à peu certains peuplements, tels ceux à Eleocharis acicularis (Duvigneaud 1983). De même le passage ou le mouillage des bateaux de pêche dans certains sites où se développent des espèces d'intérêt scientifique (Characées, Eleocharis ovata, ...) serait à réglementer. La diversité des groupements présents pourrait aussi être maintenue par un suivi de l'évolution des grands hélophytes et une gestion de ces derniers pour limiter la fermeture de certaines zones.

\section{Conclusion}

Le croisement des méthodes de traitement permet de faire apparaître des végétations stabilisées (les groupements) et des successions de végétation où les espèces pionnières (édificatrices, transgressives) ou relictuelles traduisent des relations évolutives. Nous avons pu montrer que les schémas évolutifs de la zone principale de l'étang ou de la queue de l'étang sont le résultat d'une dynamique à long terme et d'un déterminisme saisonnier. Ils traduisent en fait deux niveaux trophiques différents en lien avec le comblement. Même si l'on retrouve le fait évident que la profondeur et l'assèchement sont les facteurs déterminant la dynamique végétale de l'étang, il faut aussi remarquer qu'une dynamique de mosaïque de groupements apparaît. Il est souvent trop arbitraire de considérer que l'étang est homogène car la dynamique des étangs peut être à la fois rapide et différenciée dans l'espace, d'où la notion de zone humide complexe (Clément \& Touffet 1987). L'ensemble de l'étude nous a aussi permis de retrouver l'influence de l'aménagement et de la gestion du niveau d'eau, permettant ou non l'apparition de phytocoenoses à haute valeur patrimoniale. L'hétérogénéité du milieu, due à l'aménagement antérieur, permet de conserver des biotopes favorables à certaines de ces phytocoénoses.

\section{Travaux cités}

Amicale Phytosociologique L'. 1969. - Aperçu synoptique des unités phytosociologiques supérieures de la Brenne (Département de l'Indre, France). Bull. Soc. Bot. Fr., $116: 57-68$.

Bareau H. 1982. - Contribution à l'étude phytosociologique des étangs de la Dombes. Essai de synthèse des groupements aquatiques et subaquatiques au niveau européen. Thèse, Univ. de $\mathrm{Pa}$ -

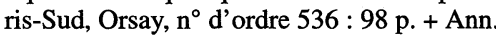


Chaib J. 1992. — Flore et végétation des milieux aquatiques et amphibies de Haute-Normandie (chorologie, phytosociologie, écologie, gestion). Thèse, Univ. de Rouen : 501 p. + Ann.

C.I.S.I.A .(Centre International de Statistique et d'Informatique Appliquées). 1991. - SPADN2.0 intégré. Saint Mandé, France.

Clément B. \& Bouzillé J.B. 1996. — La végétation des bords du lac de Grand-Lieu. 9èmes journées phytosociologiques (3-4-5 juin 1995). Bull. Soc. Bot. du Centre-Ouest, nouvelle série, 27 : 503512.

Clément B. \& Touffet J. 1987. — Typologie et diagnostic phytoécologique des zones humides de Bretagne. Colloq. Phytosoc. XV Phyto-sociologie et conservation de la nature, Strasbourg : 317$347+$ Ann.

Corillion R. 1948 (a). — Contribution à l'étude de la végétation hydrophile et hygrophile du Centre oriental armoricain. Thèse, Univ. de Toulouse : $404 \mathrm{p}$.

Corillion R. 1948 (b). — Les associations des étangs et de leurs ceintures dans le Bas-Maine Armoricain. (Végétation vasculaire, 1 ère partie). Bull. Mayenne - Sciences : 101-126.

Corillion R. 1949. - Les associations végétales des étangs du BasMaine armoricain (suite) (Associations de Charophycées, I, 1ère partie). Bull. Mayenne - Sciences : 66 - 73.

Corillion R. 1975. - Flore et Végétation du Massif Armoricain. T. IV Flore des Charophytes (Characées) du Massif Armoricain et des contrées voisines d'Europe occidentale. Edit. Jouve, Paris : $216 \mathrm{p}$.

Corillion R. 1980. — Nouvelles observations sur le dynamisme de la végétation dans les milieux aquatiques et tourbeux de l'étang de Malaguet (Chaumont d'Anjou). Mém. Soc. Et. Sci. Anjou n ${ }^{\circ} 4$ : $119-132$.

Corillion R. 1992. — Propositions pour une «liste rouge» d'espèces indigènes à protéger (Phanérogames et Cryptogames vasculaires d'Anjou). Bull. trim. Soc. Et. Sci. Anjou n ${ }^{\circ} 85$ : 19-27.

Corillion R. 1994. - Flore aquatique du Massif armoricain. Bull. de Bot. Armoricaine. E.R.I.C.A.. Conservatoire Botanique National de Brest. $\mathrm{n}^{\circ}$ 5, $103 \mathrm{p}$.

Corillion R. \& Guerlesquin M. 1965. - Evolution de la flore et de la végétation d'un étang asséché (Chaumont d'Anjou, Maine-etLoire). Bull. Soc. May.- Sci., Laval : 69 - 80.

Corillion R. \& Guerlesquin M. 1969. - Sur l'évolution récente d'une jeune tourbière neutro-alcaline à Drosera intermedia Hayne et Liparis loeselii Rich. Bull. Soc. Et. Sci. Anjou, N. S. T. VII : $135-141$.

Duvigneaud J. 1983. — La végétation des vases et graviers exondés en Lorraine française (départements de la Meurthe-et-Moselle, de la Meuse et de la Moselle) (ordre des Bidentetalia). Colloq. Phytosoc. XII. Végétations nitrophiles, Bailleul : 449 - 469.

Felzines J. C. 1981. - Les groupements du Potamion des étangs du centre de la France : aspects phytosociologiques et écologiques. Colloq. Phytosoc. X. Végétations aquatiques, Lille : 149 - 170.
Felzines J. C. 1982. - Etude dynamique, sociologique et écologique de la végétation des étangs du Centre-Est de la France. Importance de la compétition interspécifique dans l'organisation de la végétation et la distribution des espèces et des associations. Thèse, Univ. des Sciences et Techniques de Lille. $\mathrm{N}^{\circ}$ d'ordre 558 : 514 p. +5 Ann.

Felzines J. C. \& Loiseau J.- E. 1990. - Hydrophytes nouveaux ou rares de la vallée moyenne de la Loire et du Bas-Allier. Le Monde des Plantes, $\mathrm{n}^{\circ} 439: 16-19$.

Géhu J.M. \& Mériaux J.L. 1981. — Distribution et synécologie des Renoncules du sous-genre Batrachium dans le Nord de la France. Colloq. Phytosoc. X. Végétations aquatiques, Lille : 15-43.

Guinochet M. \& Vilmorin R. de. 1973-1984. - Flore de France. 5 vol. Ed. C.N.R.S., Paris : 1879 p.

Haury J. 1991. - Organisation et dynamique de la végétation d'une zone humide, aménagement et utilisation agricole pendant la période 1961-1989. Bull. Ecol. 22 (1) : 179-186.

Lambert-Servien E. 1995. - Contribution à l'étude phytoécologique des étangs de l'Anjou et de ses proches limites. Thèse, Univ. de Rennes I, $\mathbf{n}^{\circ}$ d'ordre 1512 : texte 127 p. + Ann. 113 p.

Lhote P. \& Schaefer O. 1983. - Observations phytosociologiques sur quelques étangs et bois humides du bassin de la Sereine (Bresse jurassienne). Ann. sci. de l'Univ. de Besançon, Biologie Végétale, $4^{\mathrm{e}}$ série, fasc. $4: 37-53$.

Mériaux J.-L. 1978. - Etude analytique et comparative de la végétation aquatique d'étangs et marais du Nord de la France (Vallée de la Sensée et Bassin houiller du Nord-Pas-de-Calais). Documents Phytosociologiques Nouvelle Série III, 244 p.

Mériaux J.L. 1984. — La végétation de la Somme. Colloque : L'environnement en Picardie, Amiens octobre 1984, Ed. A.M.B.E., Picardie : 81 - 91.

Mériaux J.-L. \& Wattez J.-R. 1980. — Les végétations aquatiques et subaquatiques. Relations avec la qualité des eaux. In : Pesson, P.La pollution des eaux continentales. Gauthier-Villars Ed. Paris, 2 ème éd. 225-242.

Mériaux J.-L. \& Wattez J.- R. 1981. — Groupements végétaux aquatiques et subaquatiques de la vallée de la Somme. Colloq. Phytosoc., X Végétations aquatiques, Lille : 369-413.

Oberdorfer E. 1977. - Pflanzensoziologie. Eine Reihe vegetationskundlicher Gebietsmonographien. Band 10 : Süddeutsche Pflanzengesellschaften Teil 1. Ed. VEB Gustav Fischer Verlag, Jena, $311 \mathrm{p}$.

Rallet L. 1936. - Etude phytogéographique de la Brenne. Thèse Faculté Sci. Univ. Poitiers : 293 p.

Szmeja J. \& Clément B. 1990. - Comparaison de la structure et du déterminisme des Littorelletea uniflorae en Poméranie (Pologne) et en Bretagne (France). Phytocoenologia, 19(1) : 123-148.

Touffet J. 1978. - Clefs pour l'identification préliminaire des Sphaignes armoricaines. Botanica Rhedonica, série A, $\mathrm{n}^{\circ} 16: 17$ 32. 